\title{
ORIGINAL RESEARCH \\ Temporal Bone CT Findings in Cornelia de Lange Syndrome
}

J. Kim

E.Y. Kim

J.S. Lee

W.S. Lee

H.N. Kim

BACKGROUND AND PURPOSE: Cornelia de Lange syndrome is a rare developmental malformation syndrome with a high prevalence of hearing impairment. The purposes of this study were to describe the characteristic temporal bone CT findings in patients with Cornelia de Lange syndrome and to correlate audiometric data with radiologic findings in these patients.

MATERIALS AND METHODS: Ten children (6 girls and 4 boys; mean age, 42.0 months) who were clinically diagnosed with Cornelia de Lange syndrome (classic, $n=5$; mild form, $n=5$ ) were enrolled. Temporal bone CT was prospectively performed, and 32 aspects of each temporal bone CT were analyzed, 21 by direct measurement and 11 by visual inspection. Twenty age-matched children ( $n=$ 20 ears) with normal temporal bone CT scans served as a control group. Audiologic tests were also performed on all patients.

RESULTS: Characteristic temporal bone CT findings of Cornelia de Lange syndrome were externa auditory canal stenosis, soft-tissue opacification of the hypoplastic tympanomastoid cavity, dysmorphic ossicle, hypoplastic cochlea, and dysplastic vestibule, all of which were more prevalent in patients with the classic form of the disease than in those with the mild form. Children who had more severe structural abnormalities on temporal bone CT had worse hearing levels compared with those without structural abnormalities.

CONCLUSION: Temporal bone CT scans in Cornelia de Lange syndrome could document combined structural abnormalities of the external, middle, and inner ear, which were one of the characteristic clinical manifestations; CT abnormalities were well correlated with the audiometric data.

C ornelia de Lange syndrome is a rare developmental malformation syndrome characterized by a combined congenital anomaly of multiple organs and mental retardation of unknown etiology. ${ }^{1-3}$ The characteristic craniofacial features of the highest diagnostic value include a low-set hairline in the front and back, long eyelashes, bushy eyebrows, upturned nose with anteverted nostrils, thin lips with a long philtrum, and microbrachycephaly. In addition, a variable degree of hearing impairment has been reported in $>80 \%$ of patients with Cornelia de Lange syndrome. ${ }^{1,2,4-9}$

The syndrome occurs in approximately $0.5-1$ of every 10,000 live births without any known predilection for race or sex. ${ }^{10,11}$ Most cases are usually sporadic and are thought to result from a new autosomal dominant mutation. Although a few genes, such as NIPBL and SMC1L1, are currently known to be associated with Cornelia de Lange syndrome, ${ }^{12,13}$ its diagnosis mainly depends on the recognition of the characteristic pattern of delayed growth with associated malformations, particularly the distinctive craniofacial features. ${ }^{14,15}$

Some reports have described the audiologic evaluation and histopathology of the temporal bone in children with Cornelia de Lange syndrome, ${ }^{16,17}$ but the CT findings in children with the syndrome have not been reported. Therefore, the purposes of this study were to report the characteristic findings of temporal bone

\section{Received July 6, 2007; accepted after revision October 2}

From the Department of Radiology, Research Institute of Radiological Science (J.K., E.Y.K.), and the Departments of Clinical Genetics (J.S.L.) and Otorhinolaryngology (W.S.L., H.N.K.), Yonsei University College of Medicine, Seoul, Korea.

Please address correspondence to Eung Yeop Kim, MD, Department of Radiology, Yonsei University College of Medicine, 250 Seongsanno, Seodaemun-gu, Seoul 120-752, Korea; e-mail: eungykim@yuhs.ac

Indicates article with supplemental on-line tables.

DOl 10.3174/ajnr.A0888
CT in patients with Cornelia de Lange syndrome and to correlate audiometric data with radiologic findings in these patients.

\section{Methods}

\section{Patients and Normal-Hearing Controls}

Ten children clinically diagnosed with Cornelia de Lange syndrome at a human genetics clinic were enrolled in this study for hearing impairment evaluations. Informed consent was obtained from all of the participants' parents or legal guardians, and all procedures were performed with the approval of the Institutional Review Board for Clinical Studies. The subjects consisted of 4 boys and 6 girls with ages ranging from 7 months to 8 years (mean age, 42.0 months). We categorized patients into 2 groups according to the severity of the clinical manifestations: 5 children with severe growth retardation were defined as having the classic form of the syndrome, whereas the other 5 children, who had less severe growth retardation and developmental delay, were considered as having the mild form. In addition, 20 agematched healthy controls with normal hearing $(n=20$ ears; mean age, 42.1 months) were recruited from patients who underwent a temporal bone CT examination for otitis media of the contralateral ear $(n=15)$ or minor head trauma $(n=5)$. None of the healthy controls had discernible abnormalities on temporal bone CT imaging, and there was no statistically significant difference in age between the 2 groups ( $t$ test, $P=.991$ ).

\section{CT Temporal Bone Measurements}

The temporal bone CT was prospectively performed with a 16 multidetector row CT scanner (SOMATOM Sensation 16; Siemens, Erlangen, Germany) by using a standard temporal bone protocol. Contiguous $0.7-\mathrm{mm}$ scans of the temporal bone were acquired in the axial plane and reformatted coronally with $1.0-\mathrm{mm}$ increments. CT studies of 20 ears of patients diagnosed with Cornelia de Lange syndrome and of 20 ears of controls were performed, digitally stored, and displayed 


\begin{tabular}{|c|c|c|c|c|}
\hline \multirow[b]{2}{*}{ Structure } & \multirow[b]{2}{*}{ Abnormalities } & \multicolumn{2}{|c|}{ CdL (20 ears) } & \multirow{2}{*}{$\begin{array}{l}\text { Control } \\
\text { (20 ears }\end{array}$} \\
\hline & & Classic & Mild & \\
\hline \multirow[t]{6}{*}{ Middle ear } & Ossicle dysmorphic & 10 & 2 & 0 \\
\hline & Cleft of incus body present & 8 & 2 & 0 \\
\hline & Soft-tissue opacification & 8 & 0 & 0 \\
\hline & Mastoid hypoplastic & 10 & 2 & 0 \\
\hline & Eustachian tube patulous & 0 & 0 & 0 \\
\hline & Tensor tympani absent & 0 & 0 & 0 \\
\hline \multicolumn{5}{|l|}{ Inner ear } \\
\hline \multirow[t]{2}{*}{ Cochlea } & Incomplete-partitioned & 7 & 2 & 0 \\
\hline & Modiolus deficient & 10 & 2 & 0 \\
\hline Vestibule & Focal protrusion present & 9 & 6 & 0 \\
\hline VA & Enlarged & 0 & 0 & 0 \\
\hline Facial nerve & Canal dehiscent & 3 & 0 & 0 \\
\hline
\end{tabular}

Note:-CdL indicates Cornelia de Lange syndrome; VA, vestibular aqueduct.

by using PACS (Centricity; GE Healthcare, Milwaukee, Wis). Thirty-two aspects of each temporal bone CT scan were analyzed, 11 by visual inspection and 21 by direct measurement. Two experienced head and neck radiologists who were unaware of the clinical information independently performed visual analyses of the temporal bone abnormalities. To evaluate the reliability of the measurement readings, we assessed interobserver reliability. Direct measurements were made by a head and neck radiologist by using the electronic calipers provided by the PACS. These measurements were recorded in millimeter units on the basis of the measurement method described by Purcell et al. ${ }^{18,19}$ All measurements were entered into an Excel datasheet in which averages and SDs were calculated. Data were analyzed with the Statistical Package for the Social Sciences, Version 12.0 (SPSS, Chicago, Ill), statistical software by using a $t$ test or Mann-Whitney $U$ test to compare the temporal bone measurements between patients with Cornelia de Lange syndrome and the control group. A $P$ value of .05 or less was considered significant for all analyses.

\section{Audiologic Tests}

Otologic examination and audiologic tests were performed on healthy controls as well as on all patients. Depending on the cooperation of the patients, which was largely determined by age and level of mental retardation, we performed either the play audiometry or the brainstem evoked response audiometry (BERA; GSI Audera, VIASYS Healthcare, Madison, Wis). For BERA, clicking sounds were delivered to the subjects at the rate of 33.1 clicks per second at each intensity level tested. Both ears were tested, and recordings were repeated to assess reliability. The BERA threshold was taken as the lowest stimulation level at which wave $\mathrm{V}$ could be visually detected. A BERA threshold $<40$ - $\mathrm{dB}$ normal hearing level ( $\mathrm{dB} \mathrm{nHL}$ ) was considered to be within normal limits. For children who could perform play audiometry, a threshold for both ears was calculated by averaging the thresholds at $0.5 \mathrm{kHz}, 1 \mathrm{kHz}, 2 \mathrm{kHz}$, and $4 \mathrm{kHz}$. Audiometric thresholds were correlated with temporal bone CT findings and clinical data in these patients.

\section{Results}

Measurements taken from CT scans of 20 temporal bones of patients with Cornelia de Lange syndrome were compared with measurements taken from 20 temporal bones of controls with normal hearing. CT findings determined by visual in-
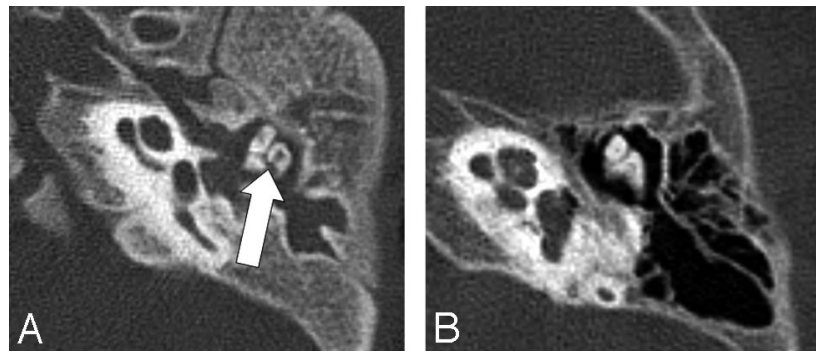

Fig 1. Axial section of the middle ear of a 2-year-old girl with Cornelia de Lange syndrome. Note the soft tissue filling the tympanomastoid cavity, the dysmorphic ossicle with a large incus body and a malleus head, and a cleft on the incus (arrow) in the patient with Cornelia de Lange syndrome $(A)$ compared with a 2-year-old control with normal hearing $(B)$.
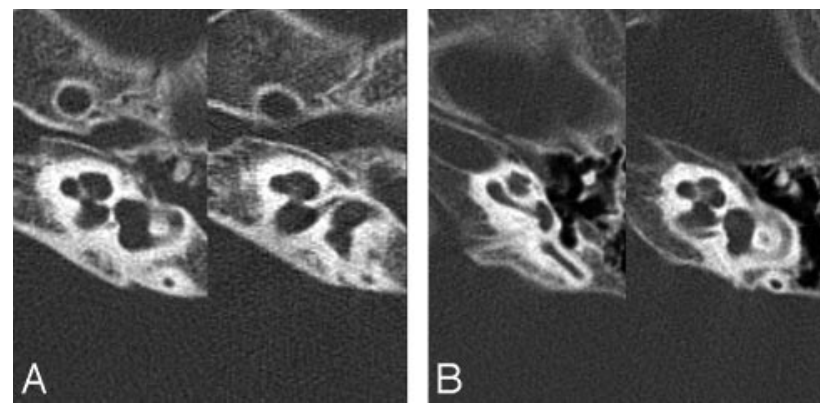

Fig 2. Axial section of the cochlea of a 4-year-old boy with Cornelia de Lange syndrome. Note the diminished width and height of cochlear upper turns with an absent modiolus in the section from the patient with Cornelia de Lange syndrome $(A)$ as compared with a 2-year-old control with normal hearing $(B)$

spection are summarized in Table 1 , and those determined by direct measurement are in the On-line Table.

Complex external, middle, and inner ear abnormalities were documented on CT scans in patients with Cornelia de Lange syndrome. Statistically significant differences between individuals with Cornelia de Lange syndrome and control groups were found in 16 of 32 categories (50\%) derived from the temporal bone. The most common and easily identifiable characteristics of Cornelia de Lange syndrome by visual inspection were the following: 1) hypoplastic mastoid $(60 \%) ; 2)$ soft-tissue opacification of the tympanomastoid cavity (40\%); 3) dysmorphism of the malleus and incus with a relatively large malleus head and an incus body (60\%) and bony cleft on the incus body (50\%) (Fig 1); 4) hypoplastic cochlea with modiolar deficiency (60\%) (Fig $2)$; 5) a bizarre-shaped vestibule with a focal protrusion at the posteroinferior aspect like a "tag" (75\%) (Fig 3); and 6) facial nerve dehiscence, mainly in the tympanic and mastoid segments (15\%). All of the aforementioned characteristics were more prevalent in patients with the classic form than in those with the mild form. The interobserver agreement for the visual detection of temporal bone abnormalities was excellent $(\kappa=0.961)$.

Analyses of the CT findings by direct measurement showed a statistically significant reduction of 8 measured values in patients with Cornelia de Lange syndrome compared with the values of the healthy controls, which included the mean luminal diameter of the external auditory canal $(P<.0001)$, bony island width of the superior semicircular canal $(P=.016)$, posterior canal lumen of the lateral semicircular canal $(P=$ .017 ), mean inferior limb length of the posterior semicircular 

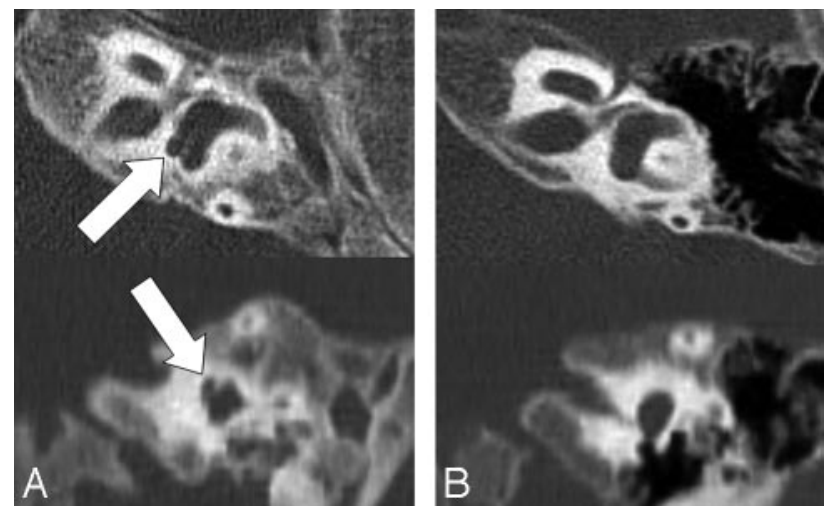

Fig 3. Axial section of a vestibule from a 2-year-old girl with Cornelia de Lange syndrome. Note the abnormal focal protrusion at the posteroinferior aspect of the vestibule (arrows) in the patient with Cornelia de Lange syndrome $(A)$ as compared with a 2-year-old control with normal hearing $(B)$.

\begin{tabular}{|c|c|c|c|c|}
\hline $\begin{array}{l}\text { Patient } \\
\text { No. }\end{array}$ & Sex/Age (yr) & $\begin{array}{l}\text { Severity of } \\
\text { Clinical } \\
\text { Features }\end{array}$ & $\begin{array}{c}\text { PA Threshold/ } \\
\text { Air-Bone Gap } \\
(\mathrm{dB} \mathrm{HL})^{*}\end{array}$ & $\begin{array}{c}\text { BERA } \\
\text { Threshold } \\
(\mathrm{dB} \text { nHL)t }\end{array}$ \\
\hline 1 & F/7 months & Classic & & R 60; L 75 \\
\hline 2 & F/8 months & Classic & & R NR; L NF \\
\hline 3 & $\mathrm{~F} / 2$ & Classic & & R 75; L 85 \\
\hline 4 & $\mathrm{M} / 3$ & Classic & & R 40; L 50 \\
\hline 5 & $\mathrm{~F} / 6$ & Classic & & R 65; L 65 \\
\hline 6 & $\mathrm{M} / 17$ months & Mild & & R 30; L 40 \\
\hline 7 & $F / 3$ & Mild & & R 20; L 20 \\
\hline 8 & $\mathrm{~F} / 4$ & Mild & & R 15; L 15 \\
\hline 9 & $\mathrm{M} / 7$ & Mild & R 30/20; L 30/20 & \\
\hline 10 & $\mathrm{M} / 8$ & Mild & R 20/5; L 20/5 & \\
\hline
\end{tabular}

Note:-PA indicates play audiometry; $R$, right ear; $L$, left ear; NR, no response. * The normal range of hearing is considered to be below $25 \mathrm{~dB}$ in play audiometry. t The normal range of hearing is considered to be below $40 \mathrm{~dB} n \mathrm{~nL}$ in BERA

canal $(P=.012)$, mean height and width of the cochlear upper turn $(P=.003$ and $P<.0001)$, and length and porus width of the internal auditory canal $(P=.002$ and $P=.003)$. However, we found no significant difference between the values in the other 13 categories.

Overall, 6 of the 10 patients in the total study population with Cornelia de Lange syndrome had hearing loss. Audiometric results showed a variable degree of mixed sensorineural and conductive hearing loss in the study (Table 2). The otoscopic examination showed abnormal drum findings in 10 of these children. Two of the 20 ears (10\%) were deaf, 8 ears showed variable degrees of hearing loss, and 8 ears had normal hearing. In 1 case of the mild form of Cornelia de Lange syndrome, serous otitis media and mild conductive hearing loss were noted.

The temporal bone CT findings were well correlated with the audiometric data. When defining the severity of structural abnormality on the basis of visual inspection per se, we found that children with more severe structural abnormalities on temporal bone CTs had worse hearing levels compared with those with fewer structural abnormalities. All patients with the classic form of the disease showed severe hearing impairments, whereas 4 patients without hearing impairments had the mild form.

\section{Discussion}

In Cornelia de Lange syndrome, the prevalence of hearing loss has been reported to be relatively high $(>80 \%)$, along with other congenital anomalies. ${ }^{4,14}$ Early diagnosis and treatment of hearing impairment is imperative in terms of language development and general behavior. Therefore, a hearing evaluation is required for pretreatment assessment of patients. ${ }^{5,14}$ Objective audiometry such as BERA is required to evaluate hearing in patients with this syndrome because of the young age and mental retardation of the patients. ${ }^{4-6}$

To our knowledge, the temporal bone CT findings in children with Cornelia de Lange syndrome have not been reported. We prospectively evaluated temporal bone CT findings associated with this syndrome despite many previous reports about hearing impairments and audiologic results. ${ }^{4-8,14}$ In our study, complex external, middle, and inner ear abnormalities were documented on CT scans in patients with Cornelia de Lange syndrome. Mean luminal diameters of the external auditory canal were significantly decreased in our patients, and Sataloff et $\mathrm{al}^{14}$ reported that stenotic external auditory canals were observed in $30 \%$ of patients with Cornelia de Lange syndrome who were included in their study. In addition, an overall decreased volume of the mastoid, a dysmorphic ossicle with a relatively large malleus head and an incus body, and facial nerve canal dehiscence were noted in our study. An unexplained bony cleft with an anteroposterior direction was present on the incus body in 10 of the 20 ears (50\%) observed in the axial scans of the temporal bone CT (Fig 1). All of the previously mentioned findings might be associated with a developmental error of the first branchial apparatus and the first pharyngeal pouch during embryogenesis as in the case of external auditory canal atresia.

In this study, we used normative measurements as well as visual inspection in the evaluation of the temporal bone, especially in the assessment of the inner ear. Subtle bony malformations may be missed because visual inspection alone is insensitive to detection. Unlike most anatomic structures, the inner ear labyrinth does not change in size after birth. ${ }^{18}$ Therefore, the establishment of normative data for the dimensions of the inner ear structures by using CT imaging will allow consistent and accurate assessment for patients of all ages. Purcell et $\mathrm{al}^{18,19}$ demonstrated that standardized measurements of inner ear structures on temporal bone CT scans can complement visual analyses and greatly aid the identification of inner ear malformations. On the basis of this premise, our prospective evaluation identified 7 different inner ear abnormalities that were statistically significant between individuals with Cornelia de Lange syndrome and those with normal hearing. Inner ear malformations observed in this syndrome consisted of hypoplastic cochlea (mainly upper turn), absent or deficient modiolus, and diminished values in some of the semicircular canal parameters, which might suggest an earlier developmental arrest than that in the middle and external ear during gestation. Although the inner ear and middle/external ear have mutually independent embryologic origins, inner ear deformities coexist in perhaps $10 \%$ of patients with the anomalies of the external and middle ear. ${ }^{20}$ As such, one would expect that comprehensive and continuous insult to the developmental ear might exist in the embryologic period as in other systemic syndromes. However, the internal auditory canal, 
which showed significantly shorter length and wider porus width in patients than in controls with normal hearing, proved to be too variable in its morphology and direction to have consistent measurements. This was reflected in the SD. Interestingly, the unusual vestibular shape was frequently observed in the patients, and a focal protruding portion at the posteroinferior aspect of the vestibule with a superomedial projection was observed like a "tag" without enlargement of the vestibular aqueduct. This unexplained finding has not been reported. Because the CT findings of Cornelia de Lange syndrome are not specific, various congenital abnormalities manifested with combined external, middle, and inner ear abnormalities should be included in the differential diagnosis_-for instance, branchio-otorenal dysplasia.

A few reports described the histopathologic findings of the temporal bone in Cornelia de Lange syndrome. ${ }^{16,17}$ Findings in these studies included short cochlea, dilated vestibule, residual mesenchyme in the perilymphatic spaces of the vestibule and semicircular canals, abnormal intrusion of vestibular ganglion cells into the facial nerve, presence of spiral ganglion cells in the internal auditory canal, loose curve of the facial nerve around the geniculum, middle ear filled with mesenchyme, and dehiscence of the facial nerve. In addition, Yamanobe and Ohtani ${ }^{16}$ described the deformed ossicle, including elongation of the anterior process of the malleus and a large incus body with a central marrow space. These findings are well correlated with our temporal bone CT findings, especially with respect to middle ear opacification, dysmorphic ossicle, hypoplastic cochlea, and facial nerve dehiscence. The characteristic radiologic findings seen on temporal bone CTs could also be considered the end result of a developmental error during the mesenchymal tissue formation, which is related to the process of membranous and perilymphatic labyrinth formation. These developmental errors have already been proved by previously described histopathologic findings. ${ }^{17}$ Our radiologic findings of the temporal bone in Cornelia de Lange syndrome should be validated by further study with a large number of cases and histopathologic correlations.

In most cases, hearing loss in Cornelia de Lange syndrome is sensorineural in origin, ranging from mild to severe, and usually bilateral. ${ }^{4-8,14}$ However, the patients in our study demonstrated middle and external ear abnormalities on their CTs. These abnormalities included maldeveloped ossicles and a diminished luminal diameter of the external auditory canal, which could contribute to conductive hearing loss in these patients. In addition, soft-tissue opacification of the tympanomastoid cavity was frequently found on temporal bone CT, which might be caused by the residual mesenchyme or superimposed otitis media. One report found that a large number of patients with Cornelia de Lange syndrome had a cleft palate or other midfacial developmental anomalies, which might also result in malfunction of the eustachian tube and middle ear effusion. ${ }^{14}$ Thus, in addition to the initial assessment for hearing impairment, hearing should be checked regularly in patients with Cornelia de Lange syndrome to detect conductive hearing loss, which is secondary to the superimposed otitis media or chronic middle ear disease.

The phenotypic pattern of Cornelia de Lange syndrome has a wide range of severity. Most patients in the first reported cases of the syndrome showed severe growth deficiency and mental retardation. However, the fact that there is a milder form of the syndrome is generally accepted as increasing numbers of more mildly affected individuals are reported. ${ }^{21,22}$ Overall, all 5 children with the clinical designation of classic Cornelia de Lange syndrome had combined inner and middle ear anomalies on their temporal bone CTs, and profound hearing loss was a characteristic feature for such patients in this study. Our findings suggest that temporal bone abnormalities may contribute to hearing loss in this syndrome and that children with more severe structural abnormalities on temporal bone CT scans show worse hearing levels. Therefore, the degree of structural abnormalities demonstrated on the temporal bone CT scans in Cornelia de Lange syndrome can predict the severity of the clinical manifestation and audiometric abnormalities as well as the prognosis for auditory development after hearing rehabilitation, as in other conditions of congenital malformation. Therefore, a CT evaluation of the temporal bone may be helpful for the pretreatment assessment of Cornelia de Lange syndrome.

\section{Conclusion}

The characteristic temporal bone CT findings for Cornelia de Lange syndrome include a stenotic external auditory canal, hypoplastic mastoid, soft-tissue opacification of the tympanomastoid cavity, dysmorphic ossicle, hypoplastic cochlea, and dysplastic vestibule, which agree with previously described histopathologic findings. Most of the patients showed a variable degree of mixed sensorineural and conductive hearing loss, and their audiometric abnormalities were well correlated with the structural abnormalities of the ear that were found on CT.

\section{References}

1. Ptacek LJ, Opitz JM, Smith DW, et al. The Cornelia de Lange syndrome. J Pediatr 1963;63:1000-20

2. Silver HK. The de Lange syndrome. Am J Dis Child 1964;108:523-29

3. Braddock SR, Lachman RS, Stoppenhagen CC, et al. Radiological features in Brachmann-de Lange syndrome. Am J Med Genet 1993;47:1006-13

4. Kaga K, Tamai F, Kitazumi E, et al. Auditory brainstem responses in children with Cornelia de Lange syndrome. Int $J$ Pediatr Otorhinolaryngol 1995;31:137-46

5. Sakai Y, Watanabe T, Kaga K. Auditory brainstem responses and usefulness of hearing aids in hearing impaired children with Cornelia de Lange syndrome. Int J Pediatr Otorhinolaryngol 2002;66:63-69

6. Ichiyama $\mathrm{T}$, Hayashi $\mathrm{T}$, Tanaka $\mathrm{H}$, et al. Hearing impairment in two boys with Cornelia de Lange syndrome. Brain Dev 1994;16:485-87

7. Egelund EP. Congenital hearing loss in patients with Cornelia de Lange syndrome (a report of two cases). J Laryngol Otol 1987;101:1276-79

8. Marres HA, Cremers CW, Jongbloet PH. Hearing levels in the Cornelia de Lange syndrome: a report of seven cases. Int J Pediatr Otorhinolaryngol 1989;18:31-37

9. McIntire MS, Eisen JD. The Cornelia de Lange syndrome: a case report with mild mental retardation. Am J Ment Defic 1965;70:438-42

10. Schuster DS, Johnson SA. Cutaneous manifestations of the Cornelia de Lange syndrome. Arch Dermatol 1966;93:702-07

11. Beck B, Fenger K. Mortality, pathological findings and causes of death in the de Lange syndrome. Acta Paediatr Scand 1985;74:765-69

12. Musio A, Selicorni A, Focarelli ML, et al. X-linked Cornelia de Lange syndrome owing to SMC1L1 mutations. Nat Genet 2006;38:528-30

13. Tonkin ET, Wang TJ, Lisgo S, et al. NIPBL, encoding a homolog of fungal Scc2-type sister chromatid cohesion proteins and fly Nipped-B, is mutated in Cornelia de Lange syndrome. Nat Genet 2004;36:636-41. Epub 2004 May 16

14. Sataloff RT, Spiegel JR, Hawkshaw M, et al. Cornelia de Lange syndrome: otolaryngologic manifestations. Arch Otolaryngol Head Neck Surg 1990;116:1044-46

15. Hawley PP, Jackson LG, Kurnit DM. Sixty-four patients with Brachmann-de Lange syndrome: a survey. Am J Med Genet 1985;20:453-59

16. Yamanobe S, Ohtani I. Temporal bone pathology in Cornelia de Lange syndrome. Otol Neurotol 2001;22:57-60

17. Sasaki T, Kaga K, Ohira Y, et al. Temporal bone and brain stem histopatholog- 
ical findings in Cornelia de Lange syndrome. Int J Pediatr Otorhinolaryngol 1996;36:195-204

18. Purcell D, Johnson J, Fischbein N, et al. Establishment of normative cochlear and vestibular measurements to aid in the diagnosis of inner ear malformations. Otolaryngol Head Neck Surg 2003;128:78-87

19. Purcell DD, Fischbein N, Lalwani AK. Identification of previously "undetectable" abnormalities of the bony labyrinth with computed tomography measurement. Laryngoscope 2003;113:1908-11
20. Mayer TE, Brueckmann H, Siegert R, et al. High-resolution CT of the temporal bone in dysplasia of the auricle and external auditory canal. AJNR Am J Neuroradiol 1997; 18:53-65

21. Van Allen MI, Filippi G, Siegel-Bartelt J, et al. Clinical variability within Brachmann-de Lange syndrome: a proposed classification system. Am J Med Genet 1993;47:947-58

22. Ireland M, Donnai D, Burn J. Brachmann-de Lange syndrome: delineation of the clinical phenotype. Am J Med Genet 1993;47:959-64 